\title{
纳米材料的化学锂化与电活性
}

\author{
麦立强 ${ }^{1,2, *}$ 杨 霜 $^{1}$ 韩春华 ${ }^{1}$ 徐 林 ${ }^{1}$ 许 絮 ${ }^{1}$ 皮玉强 ${ }^{1}$
}

('武汉理工大学材料复合新技术国家重点实验室, 武汉理工大学-哈佛大学纳米联合重点实验室, 武汉 430070;

2哈佛大学化学与化学生物系, 剑桥, 马萨诸塞州 02138, 美国)

摘要: 基于国内外最新研究进展及本课题组的研究工作, 综述了纳米材料的化学锂化与电活性研究进展. 首 先介绍了钿氧化物、钥氧化物、硒化物等高容量纳米材料的制备和锂化过程的化学问题; 然后介绍了单纳米线 器件及纳米线锂离子电池的组装、化学锂化与电活性等的最新进展. 指出单纳米线(带、管等)器件组装、锂离子 迁移原位检测、有序阵列或复杂结构设计构筑以及锂化机理、静电耦合、锂离子迁移与界面作用等相关性的研 究将是更深入探索纳米材料化学锂化与电活性的关键问题, 对纳米锂离子电池材料研究领域的发展起到促进 作用.

关键词: 锂离子电池; 电化学; 纳米材料; 化学锂化; 纳米器件

中图分类号: 0646

\section{Chemical Lithiation and Electroactivity of Nanomaterials}

\section{MAI Li-Qiang ${ }^{1,2, *} \quad$ YANG Shuang $^{1} \quad$ HAN Chun-Hua ${ }^{1} \quad X^{\prime}$ Lin $^{1} \quad X U X^{1} \quad$ PI Yu-Qiang ${ }^{1}$}

('State Key Laboratory of Advanced Technology for Materials Synthesis and Processing, WUT-Harvard Joint Nano Key Laboratory, Wuhan University of Technology, Wuhan 430070, P. R. China; ${ }^{2}$ Department of Chemistry and Chemical Biology, Harvard University, Cambridge, Massachusetts 02138, USA)

\begin{abstract}
High capacity and good cycling stability of the electrode materials are the key points to develop high-performance lithium ion battery. Based on the latest research over the world, especilly from our group, in this paper we summarized the progress in chemical lithiation and electroactivity of nanomaterials. Firstly, we introduced the preparation of high capacity nanomaterials (molybdenum oxide, vanadium oxides, selenium hydrates, etc) and the chemical problems in lithiation process. Then we summed up the progress in assembly, chemical lithiation and electroactivity of single nanowire devices and nanowire lithium ion battery. Finally, we pointed out that assembly of single nanowire (nanobelts, nanotubes, etc.) device, in situ probe of lithium ion transport, design and construction of ordered array and complex structure, investigation of lithiation mechanism, electrostatic coupling, interface interaction, etc. are effective methods to deeper exploration of the relationship between chemical lithiation and electroactivity of nanomaterials and main directions of nanoscale lithium ion battery research field.
\end{abstract}

Key Words: Lithium ion battery; Electrochemistry; Nanomaterials; Chemical lithiation; Nanodevice

\section{1 引言}

锂离子电池是当今国际公认的理想化学电源, 具有体积小、电容量大、电压高等优点, 被广泛用于
移动电话、手提电脑等电子产品, 日益扩大的电动 汽车领域将给锂离子电池带来更大的发展空间. ${ }^{1-3}$ 然而, 如何制备能量密度高、循环寿命长的锂离子

Received: February 9, 2011; Revised: April 11, 2011; Published on Web: May 19, 2011.

*Corresponding author. Email: mlq@cmliris.harvard.edu, mlq518@whut.edu.cn; Tel: +86-15717163979.

The project was supported by the National Natural Science Foundation of China (50702039, 51072153), Program for New Century Excellent Talents in Universities of the Ministry of Education of China (NCET-10-0661) and Fundamental Research Funds for the Central Universities (2010-II-016). 国家自然科学基金(50702039, 51072153), 教育部新世纪优秀人才计划(NCET-10-0661)和中央高校基本科研业务费专项基金(2010-II-016)资助

C. Editorial office of Acta Physico-Chimica Sinica 
电池一直是个挑战. 大量的研究致力于克服这些问 题, 例如掺杂、 ${ }^{4}$ 导电聚合物包覆 ${ }^{5}$ 和碳包覆 ${ }^{6}$ 等, 但是 添加导电聚合物会使电池材料的高温结构稳定性 受到影响, 而碳包覆将会降低体能量密度. 锂化被 认为是一种提高锂离子循环稳定性的有效方法. 我 们课题组最近通过单根纳米线电化学器件的设计 组装和测试, 发现电池容量的衰减与电极材料电导 率降低有关, 7 而锂化可以明显改善材料的电导率, 从而提高锂离子电池的循环稳定性. 除此之外, 通 过锂化还可研究在锂电池充放电过程中, 锂离子嵌 入和脱出对电极材料结构的影响. Johnson 等 ${ }^{8}$ 发现 采用 $\alpha-\mathrm{MnO}_{2}$ 和 $\mathrm{LiOH} \cdot \mathrm{H}_{2} \mathrm{O}$ 反应制得的 $\mathrm{MnO}_{2}$ 材料锂 化后循环时表现出比原始 $\mathrm{MnO}_{2}$ 材料更加稳定的结 构和更高的容量, 首次循环后库仑效率和循环 10 次 后容量保持率分别为 $98.2 \%$ 和 $80.2 \%$. 相对于锂化前 (75.0\%和 68.4\%), 锂化后的样品表现出更高的库仑 效率和更好的容量保持率. Landi 等 ${ }^{9}$ 在组装电池之 前碳纳米管材料电化学预锂化到 $5 \mathrm{mV}\left(v s \mathrm{Li} / \mathrm{Li}^{+}\right)$, 能够提高碳纳米管电池的循环性能. Zhang 等 ${ }^{10}$ 采用 高能球磨制得锂化的磷化钴, 与原材料相比, 锂化 的样品首次循环效率提高到 $95.0 \%$. Seong 等 ${ }^{11}$ 采用 锂化的方法大幅减小碳包覆一氧化硅的初始不可 逆容量. 锂化后, 首次充放电的库仑效率从 $67.7 \%$ 提 高到 $72.8 \%$. 他们预测, 控制用于预锂化阶段的锂含 量或者加强锂化反应将会明显提高材料的可逆容 量.

由于一维纳米材料具有特殊的化学、物理效 应, 在各个领域的研究中发现其具有许多独特的性 能. ${ }^{2-26}$ 为了进一步研究纳米尺度内材料的本征性 能, Cui 等 ${ }^{17}$ 在 $\mathrm{Si} / \mathrm{SiN}_{x}$ 基片上采用电子束刻蚀(EBL) 技术将 $\mathrm{LiMn}_{2} \mathrm{O}_{4}$ 组装成单根纳米棒器件, 通过电解 液浸泡测试其传输性能. Xue 等 ${ }^{25}$ 构筑了单根亚稳 态 $\mathrm{V}_{2} \mathrm{O}_{4} \cdot 0.25 \mathrm{H}_{2} \mathrm{O}$ 纳米线器件, 在 $-3-3 \mathrm{~V}$ 变化的偏 压下, $\mathrm{V}_{2} \mathrm{O}_{4} \cdot 0.25 \mathrm{H}_{2} \mathrm{O}$ 单根纳米线表现出非线性的 $I-V$ 特征.

层状化合物因具有典型的层状结构和良好的 锂离子嵌入与脱出特性而一直是锂离子或锂电池 材料研究的热点. 其中, 层状钒氧化物和钼氧化物 等 ${ }^{27}-35$ 容量高, 但因衰减快导致应用受到限制. 相对 于体材料而言, 纳米活性材料所具有的比表面积 大, 锂离子嵌入和脱出的距离短的特性, 使电极在 大电流下充放电极化程度小、可逆容量高、循环寿 命长. ${ }^{36-39}$ 本文基于国内外最新研究进展及本课题组
的研究工作, 综述了钼氧化物、钒氧化物、硒化物等 高比容量纳米材料的制备和锂化过程中的化学问 题, 以及纳米线锂离子电池的组装、化学锂化与电 活性等最新进展, ${ }^{40-42}$ 从而对深入理解纳米材料电化 学循环过程中的容量衰减本质及改性机制提供科 学依据和参考.

\section{2 纳米电极材料的锂化方法}

水热法通常被认为是合成高质量一维纳米材 料的一种有效方法, 例如纳米线、纳米管、纳米带 等. ${ }^{43-46}$ 最近, 我们利用水热反应成功实现纳米结构 正极材料的化学预锂化. ${ }^{40-42} \mathrm{MoO}_{3}$ 纳米带和 $\mathrm{FeSe}_{2}$ 纳 米花锂化的方法如下: (1) 水热合成 $\mathrm{MoO}_{3}$ 纳米带, 预锂化是通过与 $\mathrm{LiCl}$ 二次水热反应实现的. $\left(\mathrm{NH}_{4}\right)_{6}$ $\mathrm{Mo}_{7} \mathrm{O}_{24} \cdot 4 \mathrm{H}_{2} \mathrm{O}$ 通过离子交换树脂制得 $\mathrm{MoO}_{3} \cdot n \mathrm{H}_{2} \mathrm{O}$ 溶胶, 接着将溶胶放入聚匹氟乙烯的不锈钢反应釜 中, 在 $180^{\circ} \mathrm{C}$ 下恒温反应 $4 \mathrm{~h}$, 所得的沉淀分别用乙 醇和去离子水洗涤数次, 最后在 $80^{\circ} \mathrm{C}$ 下干燥 $8 \mathrm{~h}$, 得 到淡蓝色产物. 为了获得锂化的 $\mathrm{MoO}_{3}$ 纳米带, 将 $0.20 \mathrm{~g} \mathrm{MoO}_{3}$ 纳米带与 $0.29 \mathrm{~g} \mathrm{LiCl}$ 在去离子水中搅 拌 $2 \mathrm{~d}$ 后获得的淡蓝色溶液转移到聚四氟乙烯的不 锈钢反应釜中, 在 $180^{\circ} \mathrm{C}$ 下应 $24 \mathrm{~h}$. 在空气中冷却 后, 将得到的沉淀过滤, 用去离子水洗涤, 在 $100^{\circ} \mathrm{C}$ 下干燥. (2) 直接水热锂化 $\mathrm{MoO}_{3}$ 纳米带. 用 $40 \mathrm{~mL}$ $\mathrm{H}_{2} \mathrm{O}_{2}(30 \%)$ 与 $4 \mathrm{~g}$ 钿粉在冷水浴条件下不断地摚拌以 避免过热, 最后生成橘黄色的溶胶, 接着加入 $\mathrm{LiCl}$ 到溶胶中, 搅拌 $12 \mathrm{~h}$. 然后将此溶胶直接移入聚四氟 乙烯的不锈钢反应釜中, 在 $180^{\circ} \mathrm{C}$ 恒温 $48 \mathrm{~h}$, 自然 冷却至室温, 将所得的蓝色沉淀在 $80{ }^{\circ} \mathrm{C}$ 下干燥 4 $\mathrm{h}$, 原始的 $\mathrm{MoO}_{3}$ 纳米带在不加 $\mathrm{LiCl}$ 和其他条件不变 的情况下合成. (3) $\mathrm{FeSe}_{2}$ 纳米花同样可以通过二次 水热反应锂化. 将 $2.5 \mathrm{mmol}$ 草酸亚铁, $25 \mathrm{~mL} 0.1$ $\mathrm{mol} \cdot \mathrm{L}^{-1}$ 硒代硫酸盐溶液, $5 \mathrm{~mL} 0.1 \mathrm{~mol} \cdot \mathrm{L}^{-1}$ 柠檬酸, $25 \mathrm{~mL}$ 9.1\% (w) 聚乙烯醇溶液混合后形成均质溶 液, 将此溶液转移到聚四氟乙烯的不锈钢反应釜 中, 在 $180^{\circ} \mathrm{C}$ 下反应 $24 \mathrm{~h}$, 最后得到 $\mathrm{FeSe}_{2}$ 纳米花. 将 $\mathrm{FeSe}_{2}$ 纳米花和 $\mathrm{LiCl}$ 粉末在水中搅拌, 然后再在 $180{ }^{\circ} \mathrm{C}$ 下水热反应 $24 \mathrm{~h}$.

除了用 $\mathrm{LiCl}$ 水热锂化外, 还有其他的试剂用于 化学锂化, 例如 $\mathrm{BuLi}$ (丁基锂), $\mathrm{Li}_{2} \mathrm{~S}, \mathrm{LiI}$ 等. $\mathrm{ReO}_{3}$ 结 构的化合物也可用 $n-\mathrm{BuLi}$ 锂化, $\mathrm{ReO}_{3}$ 锂化后得到 六方复合物 $\mathrm{LiReO}_{3}$ 和 $\mathrm{Li}_{2} \mathrm{ReO}_{3}$, 这两种结构与立方 相 $\mathrm{ReO}_{3}$ 的一种扭曲结构有关. ${ }^{47,48} \mathrm{MnO}_{2}$ 能够用 $n$ - 
$\mathrm{BuLi}$ (摩尔量过量 $25 \%$ ) 或者用 $\operatorname{LiI}($ 摩尔量过量 $50 \%)$ 进行锂化. $\mathrm{Li}$ 等 49,50 比较了纯的和化学锂化的 $\mathrm{MnO}_{2}$ 的电池性能, 发现在 $370{ }^{\circ} \mathrm{C}$ 条件下, 用 $\mathrm{HNO}_{3}$ 处理的 $\mathrm{MnO}_{2}$ 和 $\mathrm{LiNO}_{3}$ 以 7:3 摩尔比反应所得到的预 锂化样品的结构稳定性提高, 锂离子扩散速度更 快, 具有更好的循环性能. Jung 等 ${ }^{51}$ 在水溶液中添加 $37 \%(w)$ 的还原剂甲醛和 $\mathrm{LiOH}$ 对 $\gamma-\mathrm{MnO}_{2}$ 进行化学 锂化, 并对得到的 $\mathrm{Li}_{x} \mathrm{MnO}_{2}$ 进行分析. 锂化溶液中的 $\gamma-\mathrm{MnO}_{2}: \mathrm{LiOH}: \mathrm{HCOH}$ 有效摩尔比为 $1: 4: 1, \mathrm{Li}_{x} \mathrm{MnO}_{2}$ 中的 $x=0.302$. 充放电测试表明锂化改善了 $\gamma-\mathrm{MnO}_{2}$ 的循环性能, 未处理过的 $\gamma-\mathrm{MnO}_{2}$ 的放电容量为 160 $\mathrm{mAh} \cdot \mathrm{g}^{-1}$, 锂化后放电容量可达 $265 \mathrm{mAh} \cdot \mathrm{g}^{-1}$.

\section{3 锂化纳米电极材料的嵌锂性能}

\section{1 锂化钼氧化物纳米材料}

如图 1A 所示, $\mathrm{MoO}_{3}$ 纳米带二次水热反应锂化 前后的 XRD 图谱的峰可以容易地归属于 $\mathrm{MoO}_{3}$ (JCPDS No.05-0508)标准谱峰. 对于未锂化的 $\mathrm{MoO}_{3}$ 纳米带, (020)、(040)和(060)峰的高强度显示纳米结 构各向异性生长以及在基片上纳米带的择优方向. 更重要的是, 与锂化前样品相比, 锂化后样品(020)
衍射峰向低角度方向有一个小的偏移, 对应于 $b$ 面 的面间距的增大, 这主要归因于层间 $\mathrm{Li}^{+}$离子的嵌 入. 用扫描电镜 (SEM) 和透射电镜(TEM) 来观察产 品的形貌和微观结构, 见图 1B. 锂化前样品的形貌 是长的带状, 宽度为 80-400 nm, 长度为 5-10 $\mu \mathrm{m}$, 可见矩形横截面. 图 $1 \mathrm{C}$ 为锂化后样品的电镜图, 在 图 1C-1 中可以清晰地看见纳米带的长度变短为 2-6 $\mu \mathrm{m}$, 有一些纳米带断裂成长度为 200-400 nm 的短片状. 在图 1C-2 中, 与锂化前的样品相比, 锂化 后的纳米带和纳米片表面变得粗糙. 入射电子束垂 直于纳米带的选区, 图 1B-3 为 $\alpha-\mathrm{MoO}_{3}$ 的(010)花样 电子衍射, 生长方向是 [001], 端/底面是(010), 侧面 是(100). 择优生长的(010)面是各向异性结构的结 果..$^{52}$

Bullard等 ${ }^{53}$ 用原子力显微镜(AFM)表征了首次 $\mathrm{Li}^{+}$嵌入的过程中 $\mathrm{MoO}_{3}(010)$ 面的结构演化. 因为 $\mathrm{Li}_{x}$ $\mathrm{MoO}_{3}$ 的晶面间距比 $\mathrm{MoO}_{3}$ 大, 所以随着它们沿(010) 方向长成 $\mathrm{MoO}_{3}$ 晶体, $\mathrm{Li}_{x} \mathrm{MoO}_{3}$ 向(010)面扩张, 如图 $1 \mathrm{C}$ 所示. 这表明锂化后的 $\mathrm{MoO}_{3}$ 晶体有大的 $b$ 面的 面间距和粗糙的表面, 这都与图 1 中的 XRD 图和 TEM 图相符合. 为了研究锂化对纳米带材料循环性
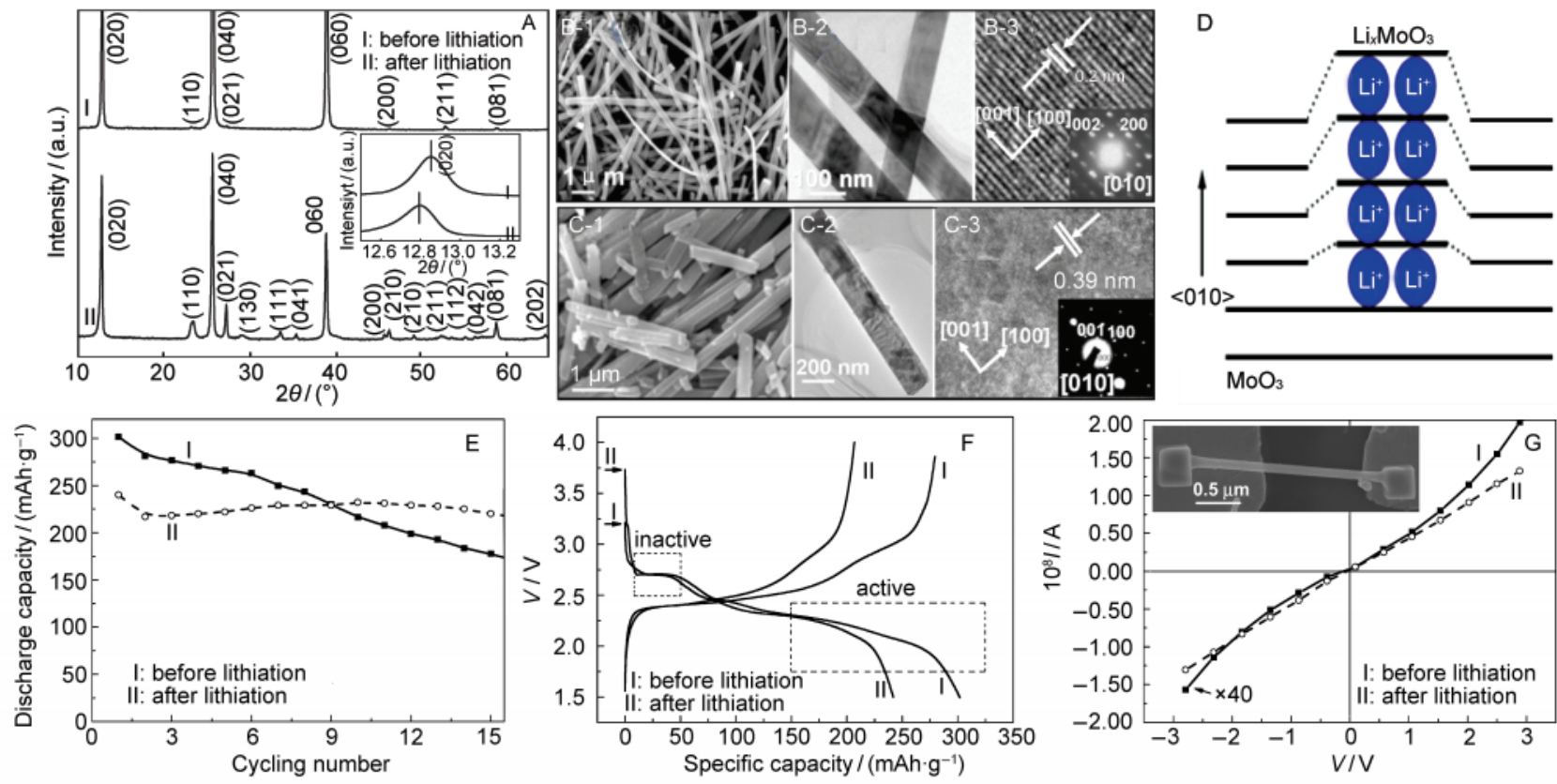

图 1 二次水热锂化前后 $\mathrm{MoO}_{3}$ 纳米带的结构表征及性能测试 ${ }^{40}$

Fig.1 Characterization and performance measurement of $\mathrm{MoO}_{3}$ nanobelts before and after lithiation via secondary hydrothermal reaction ${ }^{40}$

(A) XRD patterns of $\mathrm{MoO}_{3}$ nanobelts before and after lithiation, The inset is corresponding (020) to diffraction peak. (B, C) SEM, TEM and HRTEM images of the nanobelts before and after lithiation; The insets in the HRTEM images are corresponding to SAED patterns.

(D) schematic illustration of $\mathrm{Li}^{+}$insertion into $\mathrm{MoO}_{3}$ layers; ${ }^{35,48}$ (E) discharge capacity as a function of the cycle number for the $\mathrm{MoO}_{3}$ nanobelts before and after lithiation; (F) potential vs capacity curves for the first cycle of charge-discharge process of the nanobelts before and after lithiation; (G) $I-V$ transport measurements of single nanobelt fabricated devices using the samples before and after lithiation ${ }^{40}$ 
能的影响, 图 $1 \mathrm{E}$ 给出了锂化前后 $\mathrm{MoO}_{3}$ 纳米带在电 流密度为 $30 \mathrm{~mA} \cdot \mathrm{g}^{-1}$ 时的放电容量 - 循环次数曲线. $\mathrm{MoO}_{3}$ 纳米带的首次放电容量是 $301 \mathrm{mAh} \cdot \mathrm{g}^{-1}$, 循环 5 次后是 $246 \mathrm{mAh} \cdot \mathrm{g}^{-1}$. 而 $\mathrm{MoO}_{3}$ 体材料的首次放电 容量是 $249 \mathrm{mAh} \cdot \mathrm{g}^{-1}$, 在循环 5 次后会急剧下降到 $27 \mathrm{mAh} \cdot \mathrm{g}^{-1}$. 这可能是因为纳米带的形貌和尺寸的 影响, 使得 $\mathrm{Li}^{+}$离子扩散距离变短. ${ }^{.4}$ 我们注意到锂化 后的 $\mathrm{MoO}_{3}$ 纳米带的首次放电容量比锂化前的 $\mathrm{MoO}_{3}$ 纳米带的首次放电容量稍小. 有趣的是, $2.4 \mathrm{~V}$ 以上时, 锂化前后纳米带的首次放电比容量是相等 的, 但是在 2.4-1.5 V 时放电容量出现差异. 据报道, 锂化后的 $\mathrm{MoO}_{3}$ 在 $2.8 \mathrm{~V}$ 时发生了不可逆结构转变, 循环 2 次以后, $2.8 \mathrm{~V}$ 的平台消失, 但是锂化后在 2.4-2.0 V 可以观察到平台..$^{55}$ 换言之, 锂化后在 2.8 $\mathrm{V}$ 占位的 $\mathrm{Li}^{+}$是电化学惰性的. 因此, 根据锂化前后 纳米带的首次放电曲线(图 1F)和 XRD 图谱(图 1A), 可以推断, 一些在二次水热锂化过程中引入的 $\mathrm{Li}^{+}$占 据了一些具有储锂电化学活性的位置, 而在以后的 循环过程中, 这些 $\mathrm{Li}^{+}$被束缚在晶格中无法脱出, 从 而导致锂化后 $\mathrm{MoO}_{3}$ 纳米带的首次放电容量略小于 锂化前的. 对于未锂化的 $\mathrm{MoO}_{3}$ 纳米带, 循环 15 次后 放电容量下降至 $180 \mathrm{mAh} \cdot \mathrm{g}^{-1}$, 相应的容量保持率
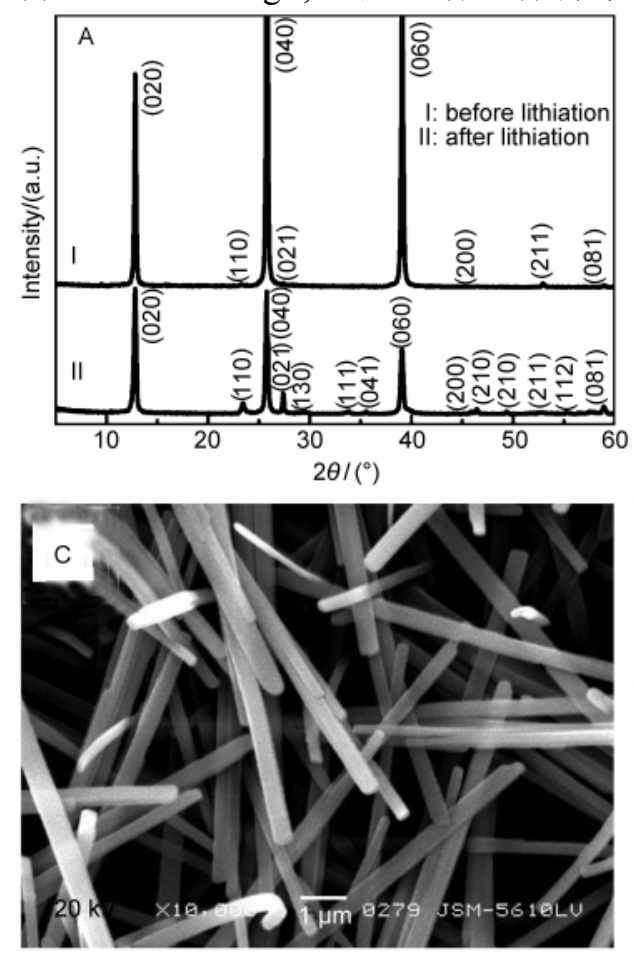

图 2 直接水热锂化前后 $\mathrm{MoO}_{3}$ 纳米带的结构表征及性能测试 ${ }^{41}$

Fig.2 Characterization and performance measurement of $\mathrm{MoO}_{3}$ nanobelts before and after lithiation via direct hydrothermal reaction ${ }^{41}$

(A) XRD patterns of $\mathrm{MoO}_{3}$ nanobelts before and after lithiation via direct hydrothermal reaction; (B) cycling property of pristine $\mathrm{MoO}_{3}$ nanobelts and directly lithiated $\mathrm{MoO}_{3}$ nanobelts; (C, D) SEM images of the nanobelts before and after lithiation, respectively ${ }^{41}$

为 $60 \%$. 而循环 15 次后, 锂化后 $\mathrm{MoO}_{3}$ 纳米带放电容 量仅下降到 $220 \mathrm{mAh} \cdot \mathrm{g}^{-1}$, 相应的容量保持率为 $92 \%$, 表现出较好的循环稳定性. 此外, Subba 等 ${ }^{56,57}$ 通过水热法制得 $\mathrm{MoO}_{3}$ 纳米带, 测得首次放电容量 为 $303 \mathrm{mAh} \cdot \mathrm{g}^{-1}$, 第 6 次循环后的放电容量为 263 $\mathrm{mAh} \cdot \mathrm{g}^{-1}$, 容量保持率为 $86.8 \%$, 第 10 次循环后的放 电容量为 $240 \mathrm{mAh} \cdot \mathrm{g}^{-1}$, 容量保持率为 $79.2 \%$. 可见, 锂化后的 $\mathrm{MoO}_{3}$ 纳米带的循环稳定性明显优于未锂 化的 $\mathrm{MoO}_{3}$ 纳米带.

除了二次水热锂化之外, 我们还研究了 $\mathrm{MoO}_{3}$ 纳米带的直接水热锂化. 图 $2 \mathrm{~A}$ 比较了锂化前后 $\mathrm{MoO}_{3}$ 纳米带的 XRD 图谱. 两种样品均为 $\alpha-\mathrm{MoO}_{3}$ (JCPDSNo.05-0508). 相对于未锂化的纳米带, (110) 和(021)峰相对强度的增加表明锂化对 $\mathrm{MoO}_{3}$ 纳米带 的结晶有一定影响, 但因为没有出现新的峰, 所以 该晶体结构仍保持正交相. 值得注意的是, 这种锂 化方法并不能扩大层间距, 这与以往所报道的不 同. ${ }^{58}$ 虽然锂离子的半径 $(60 \mathrm{pm})$ 和钼离子的半径 $(62$ $\mathrm{pm}$ )相差不大, 但是因为离子化合价的不同, 锂离子 将很难取代钼离子, 所以锂离子可能进入了 $\mathrm{MoO}_{3}$ 晶格的间隙. 图 $2 \mathrm{C}$ 和图 $2 \mathrm{D}^{41}$ 分别为锂化前后纳米带 的 SEM 图像. 锂化没有影响带状形貌, 图中还可观
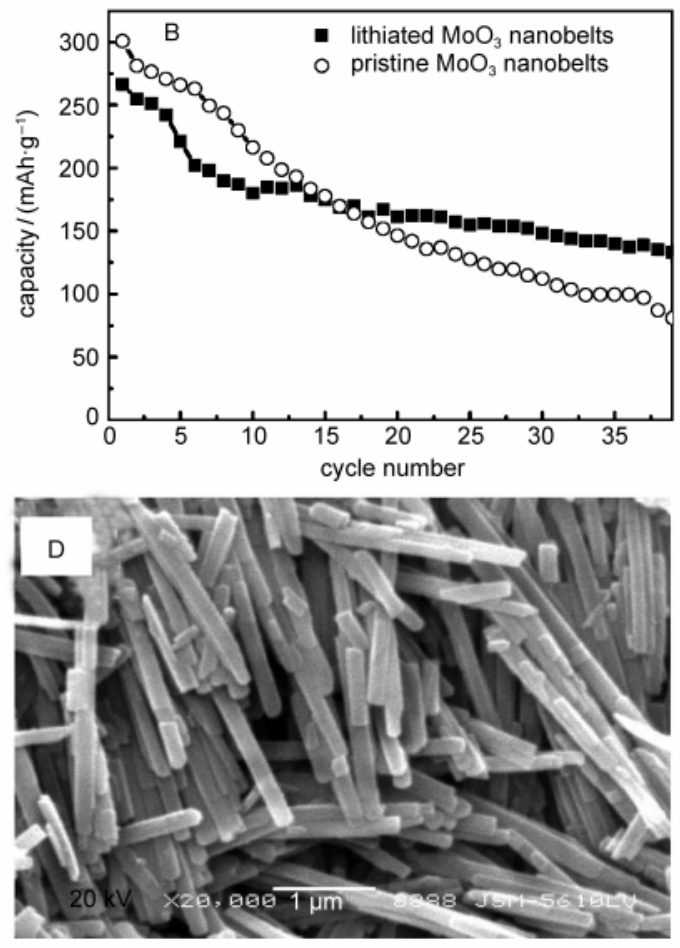
察到其矩形横截面. 但其宽度小于原始纳米带, 长 度也减小为 5-8 $\mu \mathrm{m}$, 图中还可清晰地看到长度为 200-400 nm 折断的纳米带.

图 2B 显示了锂化前后 $\mathrm{MoO}_{3}$ 纳米带电极的放 电容量-循环次数曲线. 未锂化的 $\mathrm{MoO}_{3}$ 纳米带的放 电容量随着循环次数的增加而大大降低, 从首次循 环的 $301 \mathrm{mAh} \cdot \mathrm{g}^{-1}$ 降低到第 39 次循环的 $81 \mathrm{mAh}$. $\mathrm{g}^{-1}$, 容量保持率仅为 $26.5 \%$. 锂化后 $\mathrm{MoO}_{3}$ 纳米带的 首次放电容量虽然只有 $266 \mathrm{mAh} \cdot \mathrm{g}^{-1}$, 但在第 39 次 循环后保持在 $133 \mathrm{mAh} \cdot \mathrm{g}^{-1}$, 容量保持率为 $50.0 \%$. 同样, 锂化后 $\mathrm{MoO}_{3}$ 纳米带的首次放电容量相对锂 化前有所降低, 可能归因于水热反应过程中 $\mathrm{Li}^{+}$占据 了一些具有储锂电化学活性的间隙位置. 锂化后纳 米带具有更好的循环稳定性, 一方面归因于锂离子 的嵌入对 $\mathrm{MoO}_{3}$ 层结构起到了支撑作用, 使八面体 在电化学循环中因锂离子嵌入与脱出导致体积变 化减小, 另一方面可能因为锂化过程锂离子的嵌入 提高了材料载流子浓度和电导率. ${ }^{41}$

\section{2 锂化钒氧化物纳米材料}

Cui 等 ${ }^{59}$ 研究了采用 $\mathrm{BuLi}$ 锂化单根 $\mathrm{V}_{2} \mathrm{O}_{5}$ 纳米 带，发现锂化后生成 $\mathrm{Li}_{x} \mathrm{~V}_{2} \mathrm{O}_{5}$, 即 $\omega-\mathrm{Li}_{3} \mathrm{~V}_{2} \mathrm{O}_{5}$ 、 $\gamma-\mathrm{Li}_{2} \mathrm{~V}_{2} \mathrm{O}_{5}$. 并用 $\mathrm{Br}_{2}$ 处理锂化后的样品, 得到完全去 锂化的 $\mathrm{V}_{2} \mathrm{O}_{5}$. 锂离子的嵌入和脱出过程的可逆性对 研究锂离子二次电池至关重要. 通过 TEM 表征得 出, 去锂化是通过下述反应实现的:

\section{$\mathrm{Li}_{x} \mathrm{~V}_{2} \mathrm{O}_{5}+0.5 x \mathrm{Br}_{2} \rightarrow \mathrm{V}_{2} \mathrm{O}_{5}+x \mathrm{LiBr}$}

结果表明, 锂化后的 $\mathrm{V}_{2} \mathrm{O}_{5}$ 纳米带能够去锂化形 成与原始材料一样的单晶正交相 $\mathrm{V}_{2} \mathrm{O}_{5}$, 而对于 $\omega-\mathrm{Li}_{3} \mathrm{~V}_{2} \mathrm{O}_{5}$, 去锂化是完全可逆的. 电化学去锂化一 直局限于 $\omega-\mathrm{Li}_{3} \mathrm{~V}_{2} \mathrm{O}_{5}$, 去锂化后, 锂含量仍保持在 $x=$
0.4, 而用 $\mathrm{Br}_{2}$ 对块体的 $\mathrm{V}_{2} \mathrm{O}_{5}$ 进行去锂化后, 可以使锂 含量减小到 $x=0.1$, 但是去锂化后的材料为立方结 构.

为了进一步表征锂化前后化学成分的变化, 采 用电子能量损失谱(EELS)对锂化前后的样品进行 测试. 由图 3(A, B) 可知, 锂化后的纳米带中有锂存 在, 同时根据扫描透射电镜(STEM)-EELS 结果(图 3 $(\mathrm{C}, \mathrm{D}))$ 可知, 锂离子浓度是不均匀的, 在边界部分由 于可以直接与 $\mathrm{BuLi}$ 接触而浓度较高, 同时由图 $3 \mathrm{C}$ 虚线区域可知, 在纳米带表面缺陷处也具有较高的 锂离子浓度(图3D中亮度越高代表锂离子浓度越 高), 而这同样是因为缺陷处直接与 $\mathrm{BuLi}$ 接触的缘 故. 这些都与纳米尺度内大的界面接触面积所导致 锂离子嵌入距离变短的结论相符合.

Delmasa等 ${ }^{60}$ 的实验结果表明, 嵌入锂后的 $\mathrm{V}_{2} \mathrm{O}_{5}$ 纳米带可以完全去锂化, 锂化后保持单晶结构完 整, 基于 $\mathrm{V}_{2} \mathrm{O}_{5}$ 放电曲线, 可逆容量增加了 $17.0 \%$, 并 扩展截止电压范围到 $4 \mathrm{~V}$, 能量密度经估算增加了 $30.0 \%$.

Garcia 等 ${ }^{61}$ 研究了用化学方法预锂化 $\mathrm{V}_{2} \mathrm{O}_{5}$, 发 现预锂化可以大大影响 $\mathrm{V}_{2} \mathrm{O}_{5}$ 的电化学性能. 在氩气 气氛下以相应的化学计量比向 $\mathrm{V}_{2} \mathrm{O}_{5}$ 粉末中滴入正 丁基锂溶液 $\left(1.6 \mathrm{~mol} \cdot \mathrm{L}^{-1}\right.$ 正己烷)可以锂化 $\mathrm{V}_{2} \mathrm{O}_{5}$, 得 到 $\mathrm{Li}_{x} \mathrm{~V}_{2} \mathrm{O}_{5}$. 反应如下:

$$
\mathrm{V}_{2} \mathrm{O}_{5}+x \mathrm{C}_{4} \mathrm{H}_{9} \mathrm{Li} \rightarrow \mathrm{Li}_{x} \mathrm{~V}_{2} \mathrm{O}_{5}+x / 2 \mathrm{C}_{8} \mathrm{H}_{18}
$$

他们得到三种 $\mathrm{Li}_{x} \mathrm{~V}_{2} \mathrm{O}_{5}$ 化合物, 分别为 $\mathrm{Li}_{0.9} \mathrm{~V}_{2} \mathrm{O}_{5}$ 、 $\mathrm{Li}_{1.16} \mathrm{~V}_{2} \mathrm{O}_{5}$ 和 $\mathrm{Li}_{1.6} \mathrm{~V}_{2} \mathrm{O}_{5}$. 在首次充电过程中, 这三种锂 化样品中锂离子的脱出都不完全, 存在以下两种可 能: (1) 预锂化过程所引入的锂离子被束缚在晶格 中; (2) 用氧气对 $\mathrm{Li}_{x} \mathrm{~V}_{2} \mathrm{O}_{5}$ 化合物进行的化学氧化可
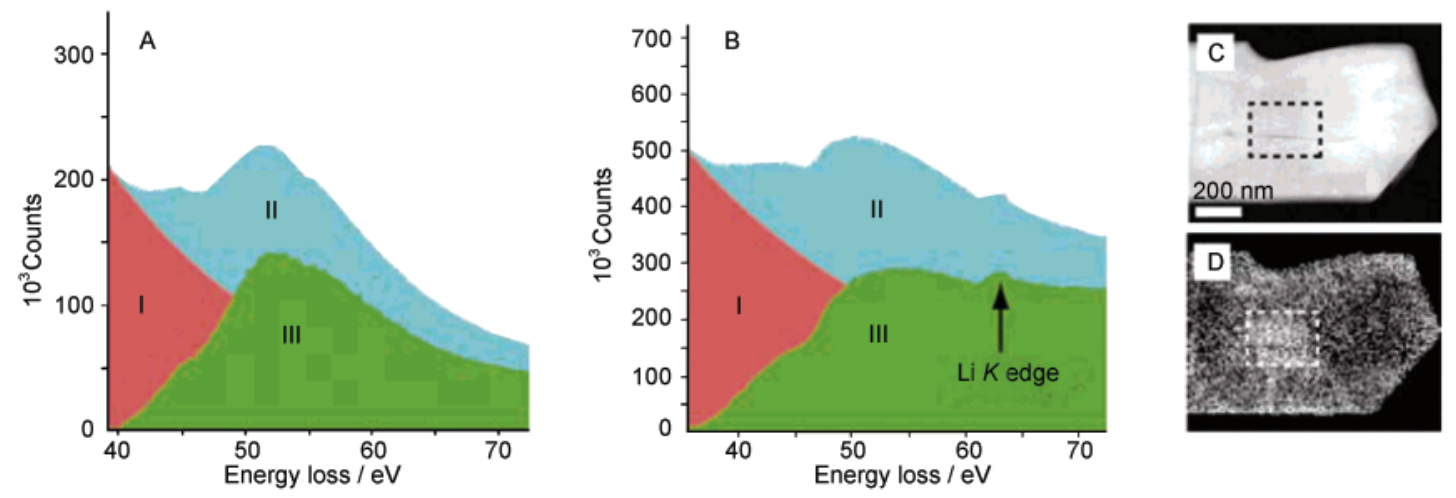

图 3 锂化前后 $\mathrm{V}_{2} \mathrm{O}_{5}$ 纳米带的结构表征测试 ${ }^{59}$

Fig.3 Characterization measurement of $\mathrm{V}_{2} \mathrm{O}_{5}$ nanobelts before and after lithiation ${ }^{59}$

(A-B) electron energy loss spectroscopy (EELS) spectra of nanobelts with background extrapolated using log polynomial law fitting. The I is the background, the II is the total signal, and the III is extracted nanobelt signal. (C) Scanning transmission electron microscope (STEM) image of $\mathrm{Li}_{\mathrm{x}} \mathrm{V}_{2} \mathrm{O}_{5}$ nanobelt with a dashed box indicating a crack in the structure. (D) Corresponding EELS spectrum image (same scale as Fig.3C) ${ }^{59}$ 
以解释在充电过程中发现低的感应电流产生, 而这 个化学氧化可能导致在化合物的表面形成其它晶 相, 如 $\mathrm{Li}_{2} \mathrm{CO}_{3}$ 或 $\mathrm{LiOH}$. 在随后的放电过程中, 锂化材 料中嵌入的锂离子总量几乎和原始的氧化物相同. 有意思的是, 对于锂化后的样品而言, 循环稳定性 和陈化时间有关. 20 个循环以后, 经过 11 天和 48 天 陈化的样品其比容量大约为 $280-300 \mathrm{mAh} \cdot \mathrm{g}^{-1}$, 而 刚锂化的 $\mathrm{Li}_{1.16} \mathrm{~V}_{2} \mathrm{O}_{5}$ 和 $\mathrm{V}_{2} \mathrm{O}_{5}$ 的比容量为 $250 \mathrm{mAh}$. $\mathrm{g}^{-1}$, 这表明陈化后容量增加了 $15 \%-20 \%$. 化学氧化 所造成的形貌及其结构的改变导致了非晶化合物 的产生, 从而得到更好的循环性能. Lee 等 ${ }^{62}$ 利用 $\mathrm{XRD}$ 以及原位拉曼测试技术发现, 在锂离子嵌入过 程中发现电极材料非晶化, 同时伴随有较大的体积 膨胀. 尽管高的可逆容量伴随着大的体积膨胀, 但 无序结构的存在一定程度上使得纳米尺度内的电 极材料能够容纳这种体积变化, 从而使电极材料结 构得到完整的保存, 即使在深度充放电下, 纳米颗 粒依然具有较好的循环性能. 众所周知, 过大的体 积膨胀会造成电极材料结构的崩塌, 最终导致电池 的寿命减短. 然而局部结构的变化, 即非晶化结构
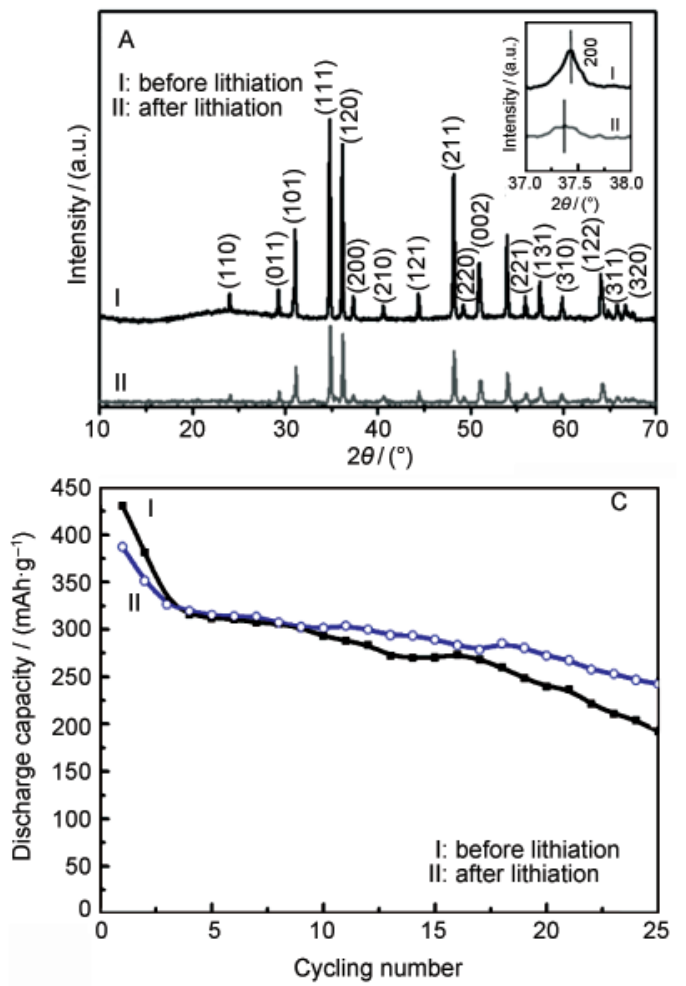

的存在可以在一定程度上缓解锂离子嵌入和脱出 过程中造成的较大体积变化对电极材料结构的影 响, 从而较好地保持了材料结构的完整性, 提高了 材料的循环稳定性.

\section{3 锂化硒化亚铁纳米材料}

为了进一步研究化学预锂化对正极材料循环 性能的影响, 我们选择了典型的半导体 $\mathrm{FeSe}_{2}$ 作为另 一个例子. 值得注意的是, 铁基化合物作为锂离子 电池的电极材料是具有优势的, 因为铁很丰富、价 格便宜而且具有环境友好性. ${ }^{63-65}$ 图 4A 为锂化前后 $\mathrm{FeSe}_{2}$ 纳米花的 XRD 图谱. 所有的衍射峰都与 $\mathrm{FeSe}_{2}$ 相匹配(JCPDS No.82-0269). 相对于未锂化样品, 锂 化样品中的(200)峰有一个向低衍射角的微小转变, 见图 4A 插图. 这一结果表明锂化后 $a$ 面的面间距有 所扩大, 这可能是由于锂填隙原子的引入所导致. 图 4B 是以上所制备的 $\mathrm{FeSe}_{2}$ 纳米花的场发射扫描电 镜(FESEM)图像, 可以看出, 纳米花由均匀的纳米片 组成, 这些纳米片的厚度约为 $20 \mathrm{~nm}$, 直径约为 100 nm.

图 $4 \mathrm{C}$ 是在锂化前后 $\mathrm{FeSe}_{2}$ 纳米花的放电容量-
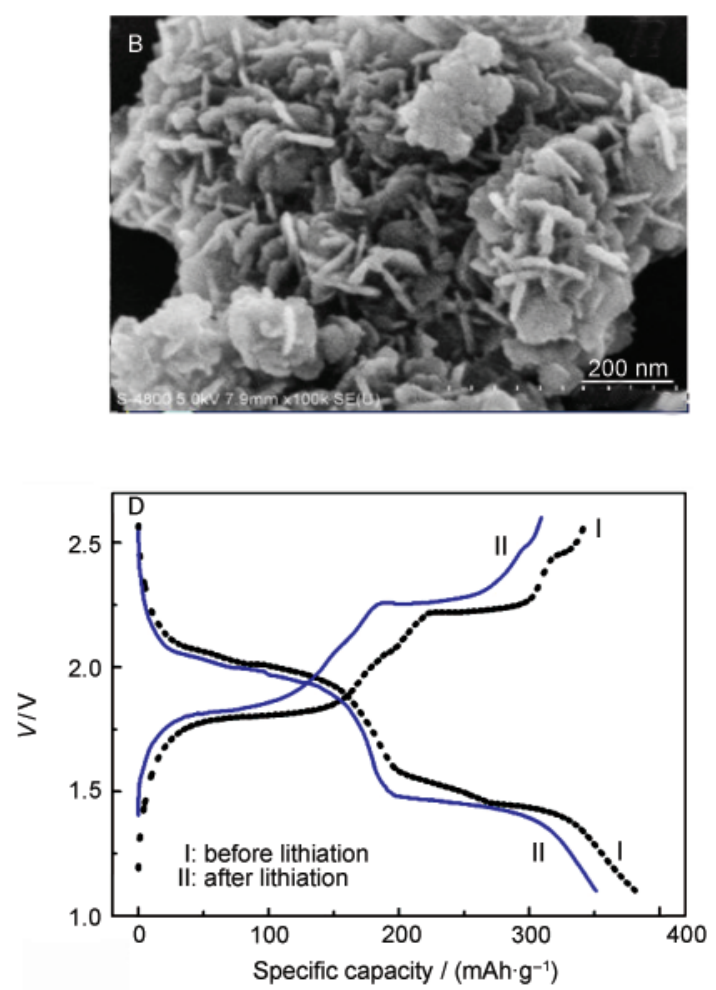

图 4 锂化前后 $\mathrm{FeSe}_{2}$ 纳米花的结构表征及性能测试 ${ }^{42}$

Fig.4 Characterization and performance measurement of $\mathrm{FeSe}_{2}$ nanoflowers before and after lithiation ${ }^{42}$ (A) XRD patterns of $\mathrm{FeSe}_{2}$ nanoflowers before and after lithiation; (B) FESEM image of the as-prepared $\mathrm{FeSe}_{2}$ nanoflowers; (C) The discharge capacity as a function of the cycle number for the $\mathrm{FeSe}_{2}$ nanoflowers before and after lithiation; (D) potential vs capacity curves for the second cycle of charge-discharge process of the nanoflowers before and after lithiation ${ }^{42}$ 
循环次数曲线, 电流密度为 $40 \mathrm{~mA} \cdot \mathrm{g}^{-1}$. 未锂化的 $\mathrm{FeSe}_{2}$ 纳米花第一次和第二次的放电容量分别达到了 431 和 $382 \mathrm{mAh} \cdot \mathrm{g}^{-1}$, 在第一次循环中有大约 49 $\mathrm{mAh} \cdot \mathrm{g}^{-1}$ 的不可逆容量. 而锂化的纳米花在第一次循 环中大约只有 $36 \mathrm{mAh} \cdot \mathrm{g}^{-1}$ 的不可逆容量. 经过 25 个 循环以后, 未锂化的 $\mathrm{FeSe}_{2}$ 纳米花其放电容量减少到 了 $192 \mathrm{mAh} \cdot \mathrm{g}^{-1}$, 只有初始容量的 $45.0 \%$. 然而, 经过 25 次循环以后, 锂化的 $\mathrm{FeSe}_{2}$ 纳米花的放电容量为 $242 \mathrm{mAh} \cdot \mathrm{g}^{-1}$, 为初始容量的 $63.0 \%$, 这表明锂化后的 纳米花循环性能得到了提高. 图 4D 为锂化前后 $\mathrm{FeSe}_{2}$ 纳米花在第二次循环中的电压-容量关系曲线, ${ }^{42}$ 由 图可知, 锂化后 $\mathrm{FeSe}_{2}$ 纳米花的首次放电容量小于锂 化前的纳米花. 根据图 4A 中的 XRD 数据可以推断, 这可能是因为在二次水热锂化过程中引入的一些锂 离子占据了具有储锂电化学活性的空隙位置. 锂离 子在纳米花中的预嵌入极大地改善了循环稳定性和 可逆性, 而占据 $\mathrm{FeSe}_{2}$ 晶格空隙的锂离子可以使结构 更加稳定并减少了放电过程中 $\mathrm{FeSe}_{2}$ 层和层间锂离 子的静电作用. ${ }^{4}$

\section{4 纳米器件与电活性}

我们课题组采用静电纺丝技术设计制备出具 有 “线中棒” 分级结构的钒氧化物超长纳米线, 发现
该纳米线作为锂离子电池正极材料具有高的比容 量和优异的循环性能. ${ }^{6}$ 相对于常规纳米材料, 这种 新颖的分级纳米结构可以有效避免纳米材料因具 有高的比表面能而容易发生自团聚现象, 从而提高 电池的性能. 为了排除纳米材料团聚对性能的影 响, 我们设计单根纳米线的电化学器件, 通过原位 表征, 建立了纳米线的电输运、结构与电极充放电 状态的直接联系, 发现容量衰减与电导率的降低有 关. 为了进一步研究化学锂化对纳米材料本征电活 性的影响, 我们课题组将锂化前后单根 $\mathrm{MoO}_{3}$ 纳米 带组装成纳米器件, 测试其电输运性能(图1G). 锂 化前, $I-V$ 特性显示出纳米带两端的不对称肖特基 势垒(图 $1 \mathrm{G}$ 中的实线), 这是在半导体氧化钼(带隙 为 $3.1 \mathrm{eV}$ ) 和金/铂电极之间产生的, 在大约 $2 \mathrm{~V}$ 时, 传输电流约为 $300 \mathrm{pA}$. 锂化后, $I-V$ 曲线显示出欧姆 特性(图 $1 \mathrm{G}$ 中的虚线), 在大约 $2 \mathrm{~V}$ 时, 传输电流约为 $10 \mathrm{nA}$. 根据测定的电阻、有效长度和横截面积计 算, 纳米带锂化前后的电导率大约分别为 $10^{-4}$ 和 $10^{-2} \mathrm{~S} \cdot \mathrm{cm}^{-1}$. 通过锂化, 电导率增加了近两个数量 级. 因为纳米带沿(001)面生长, 所以纳米带导电性 的增加意味着 $\mathrm{MoO}_{6}$ 八面体层中载流子浓度的增 加, 而这表明锂离子是作为填隙离子被引入的, 在
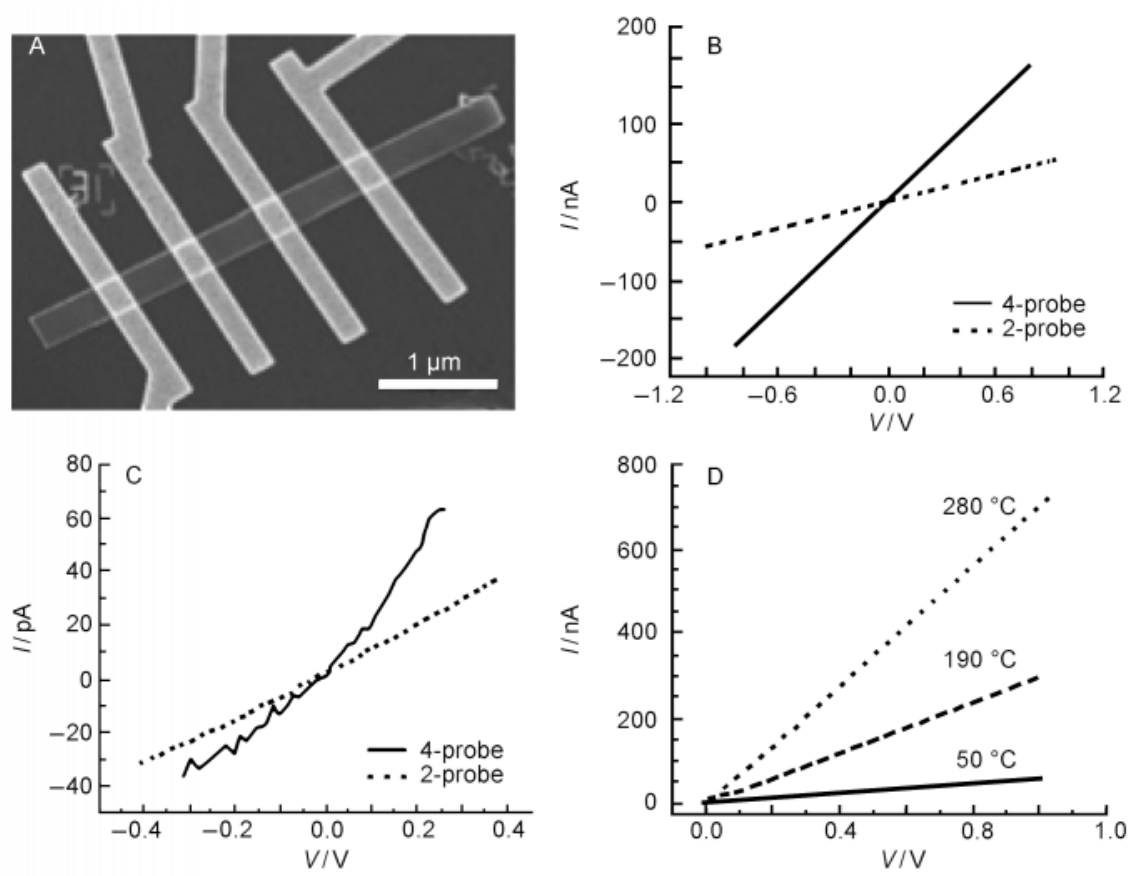

图 5 单根 $\mathrm{Li}_{x} \mathrm{~V}_{2} \mathrm{O}_{5}$ 纳米带器件及 $I-V$ 曲线测试 ${ }^{59}$

Fig.5 Single $\mathrm{Li}_{x} \mathrm{~V}_{2} \mathrm{O}_{5}$ nanobelt device and $I-V$ characteristics

(A) SEM image of single nanobelt contacted by four metal electrodes; (B-D) current-voltage characteristics of individual nanobelts; (B, C) measurements performed on the same nanobelt; (B) two- and four-probe $I-V$ characteristics for pristine $\mathrm{V}_{2} \mathrm{O}_{5}$ nanobelt; (C) two and four-probe $I-V$ characteristics for $\mathrm{Li}_{x} \mathrm{~V}_{2} \mathrm{O}_{5}$ nanobelt treated with BuLi for $2 \mathrm{~h}$; (D) $I-V$ characteristics for pristine $\mathrm{V}_{2} \mathrm{O}_{5}$ nanobelt at different temperatures, showing the nanobelt being stable at high temperatures ${ }^{59}$ 
电化学循环过程中, $\mathrm{MoO}_{3}$ 的层间距随着锂离子的嵌 入或脱出而不断扩大或缩小. 相对于未锂化样品, 具有较宽层间距的锂化后 $\mathrm{MoO}_{3}$ 纳米带在充放电过 程中显示出了较小的体积变化. 因此, 锂化可以提 高 $\mathrm{MoO}_{3}$ 电极在锂离子嵌入和脱出过程中的结构稳 定性. 首次锂化所引入的锂离子保留在晶格中, 从 而提高了导电性, 这将有助于未来充放电过程中锂 离子的嵌入和脱出. 这一结果与前面锂化后 $\mathrm{MoO}_{3}$ 纳米带循环稳定性提高相一致.

与层状 $\mathrm{MoO}_{3}$ 纳米带一样, 另外一种典型的层 状正极材料为 $\mathrm{V}_{2} \mathrm{O}_{5}$, 因其具有高的嵌锂容量一直是 锂离子电池材料研究的热点. Cui 等 ${ }^{59}$ 将其制得的锂 化前后的 $\mathrm{V}_{2} \mathrm{O}_{5}$ 纳米带组装成纳米器件研究了锂化 对 $\mathrm{V}_{2} \mathrm{O}_{5}$ 本征电化学性能的影响, 测量了锂化前单根 纳米带的电输运性能. 采用四电极法测得单根纳米 带的 $I-V$ 曲线 (见图 5). 由图 5 可知, $I-V$ 曲线是线性 对称的, 两探针间电阻为 $9.50 \mathrm{M} \Omega$, 为了得到单根纳 米带的本征电阻, 采用四探针测量, 得到材料的本 征电阻为 $9.04 \mathrm{M} \Omega$, 接触电阻为 $0.46 \mathrm{M} \Omega$, 通过对大 约 20 根单根纳米带的测量得到材料的本征电导率 为 $0.8-3.4 \mathrm{~S} \cdot \mathrm{cm}^{-1}$. 这比单晶体材料的电导率 $(0.02$ $\left.\mathrm{S} \cdot \mathrm{cm}^{-1}\right)$ 高得多, 可能的原因是纳米带的高结晶度改 善了纳米带中载流子的输运路径. 同样, 对锂化后 的单根纳米带的电输运性能进行测量, 得其电阻为 $6.30 \mathrm{M} \Omega$, 接触电阻为 $3.40 \mathrm{M} \Omega$, 单根 $\mathrm{Li}_{x} \mathrm{~V}_{2} \mathrm{O}_{5}$ 纳米带 的本征电导率为 $0.001 \mathrm{~s} \cdot \mathrm{cm}^{-1}$, 比原始 $\mathrm{V}_{2} \mathrm{O}_{5}$ 纳米带 低 3 个数量级. 与锂化的 $\mathrm{MoO}_{3}$ 纳米带不同的是, 锂 化后的 $\mathrm{V}_{2} \mathrm{O}_{5}$ 纳米带是混合相, 即 $\omega-\mathrm{Li}_{3} \mathrm{~V}_{2} \mathrm{O}_{5}$ 、 $\gamma-\mathrm{Li}_{2} \mathrm{~V}_{2} \mathrm{O}_{5}$, 而混合相所产生的电子传导势垒或者局 部电子结构的变化可能导致低的电导率和高的接 触电阻. 尽管电导率低, 但是 $I-V$ 曲线保持线性, 在-6-6V之间稳定地循环. 如果只考虑电输运, 据 估计, 在 $4 \mathrm{~V}$ 时只需要少于 $4.5 \mathrm{~s}$ 的时间为单根纳米 带充电, 这将非常适于高能电池的应用.

\section{5 结语与展望}

化学锂化纳米结构既包含了纳米结构应有的 优点(和块体材料相比), 又包含了预锂化的优点(和 未锂化的样品相比), 对于研制高性能锂离子电池具 有重要意义. 为了更深入探索纳米材料化学锂化与 电活性关系以及如何有效抑制锂离子电池的容 量衰减, 需要开展单纳米线(带、管等)器件组装、7 锂离子迁移原位检测、 ${ }^{13,24,67}$ 有序阵列或复杂结构构
筑 ${ }^{66,68,69}$ 以及锂化机理、静电耦合、锂离子迁移与界面 作用等关键化学问题的研究, 这必将是纳米锂离子 电池材料研究领域的重要发展方向, 并已开始引起 人们的关注.

致谢: 非常感谢美国西北太平洋国家实验室 J. Liu研究员、 哈佛大学 C. M. Lieber 教授及佐治亚理工大学 Z. L. Wang 教 授、武汉理工大学陈文教授的讨论、合作和大力帮助. 同时感 谢哈佛大学的Y. J. Dong 博士、麻省理工学院Y. Shao 教授、 加州大学伯克利分校 C. S. Lao 博士、牛津大学 Y. Z. Huang 博士、武汉理工大学韩久慧等的合作与帮助.

\section{References}

(1) Goodenough, J. B. J. Power Sources 2007, 174, 996.

(2) Ma, M.; Chernova, N. A.; Toby, B. H.; Zavalij, P. Y.; Whittingham, M. S. J. Power Sources 2007, 165, 517.

(3) Ji, X.; Lee, T.; Nazar, L. F. Nat. Mater. 2009, 8, 500.

(4) Lee, Y.; Kim, M. G.; Cho, J. Nano Lett. 2008, 8, 957.

(5) Huang, X. H.; Tu, J. P.; Xia, X. H.; Wang, X. L.; Xiang, J. Y. Electrochem. Commun. 2008, 10, 1288.

(6) Doherty, C. M.; Caruso, R. A.; Smarsly, B. M.; Adelhelm, P.; Drummond, C. J. Chem. Mater. 2009, 21, 5300.

(7) Mai, L. Q.; Dong, Y. J.; Xu, L.; Han, C. H. Nano Lett. 2010, 10, 4273.

(8) Johnson, C. S.; Dees, D. W.; Mansuetto, M. F.; Thackeray, M. M.; Vissers, D. R. J. Power Sources 1997, 68, 570.

(9) Landi, B. J.; Ganter, M. J.; Cress, C. D.; DiLeo, R. A.; Raffaelle, R. P. Energy Environ. Sci. 2009, 2, 638.

(10) Zhang, Z.; Yang, J.; Nuli, Y.; Wang, B.; Xu, J. Solid State Ionics 2005, 176, 693.

(11) Seong, I. W.; Kim, K. T.; Yoon, W. Y. J. Power Sources 2009, 189, 511.

(12) Tian, B.; Cohen-Karni, T.; Qing, Q.; Duan, X.; Xie, P.; Lieber, C. M. Science 2010, 329, 830.

(13) Huang, J. Y.; Zhong, L.; Wang, C. M.; Sullivan, J. P.; Xu, W.; Zhang, L. Q.; Mao, S.; Hudak, N.; Liu, X. H.; Subramanian, A. K.; Fan, H.; Qi, L.; Kushima, A.; Li, J. Science 2010, 330, 1515.

(14) Tian, B.; Zheng, X.; Kempa, T. J.; Fang, Y.; Yu, N.; Yu, G.; Huang, J.; Lieber, C. M. Nature 2007, 449, 885.

(15) Dong, Y. J.; Yu, G. H.; McAlpine, M. C.; Lu, W.; Lieber, C. M. Nano Lett. 2008, 8, 386.

(16) Kempa, T. J.; Tian, B. Z.; Kim, D. R.; Hu, J. S.; Zheng, X. L.; Lieber, C. M. Nano Lett. 2008, 8, 3456.

(17) Yang, Y.; Xie, C.; Ruffo, R.; Peng, H. L.; Kim, D. K.; Cui, Y. Nano Lett. 2009, 9, 4109.

(18) Schoen, D. T.; Peng, H. L.; Cui, Y. J. Am. Chem. Soc. 2009, 131, 7973.

(19) Cha, J. J.; Williams, J. R.; Kong, D. S.; Meister, S.; Peng, H. L.; Bestwick, A. J.; Gallagher, P.; Gordon, D. G.; Cui, Y. Nano Lett. 2010, 10, 1076.

(20) Hu, Y. F.; Chang, Y. L.; Fei, P.; Snyder, R. L.; Wang, Z. L. ACS 
Nano 2010, 4 (2), 1234.

(21) Xu, S.; Qin, Y.; Xu, C.; Wei, Y. G.; Yang, R. S.; Wang, Z. L. Nat. Nanotechnol. 2010, 5, 366.

(22) Baik, J. M.; Kim, M. H.; Larson, C.; Yavuz, C. T.; Stucky, G. D.; Wodtke, A. M.; Martin, M. Nano Lett. 2009, 9, 3980.

(23) Tian, B.; Xie, P.; Kempa, T. J.; Bell, D. C.; Lieber, C. M. Nat. Nanotechnol. 2009, 4, 824.

(24) Wang, C. M.; Xu, W.; Liu, J.; Choi, D.; Arey, B. W.; Saraf, L. V.; Zhang, J.; Yang, Z.; Thevuthasan, S.; Baer, D. R.; Salmon, N. J. Mater. Res. 2010, 25, 1541.

(25) Xue, X. Y.; Feng, P.; Wang, C.; Chen, Y. J.; Wang, Y. G.; Wang, T. H. Appl. Phys. Lett. 2006, 89, 022115.

(26) Schoe, D. T.; Xie, C.; Cui, Y. J. Am. Chem. Soc. 2007, 129, 4116.

(27) Mai, L. Q.; Chen, W.; Xu, Q.; Zhu, Q. Y.; Han, C. H.; Peng, J. F.; Solid State Commun. 2003, 126, 541.

(28) Qi, Y. Y.; Chen, W.; Mai, L. Q.; Zhu, Q. Y.; Jin, A. P. Int. J. Electrochem. Sci. 2006, 1, 317.

(29) Chen, W.; Mai, L. Q.; Qi, Y. Y.; Dai, Y. J. Phys. Chem. Solids 2006, 67, 896.

(30) Chernova, N. A.; Roppolo, M.; Dillon, A. C.; Whittingham, M. S. J. Mater. Chem. 2009, 19(17), 2526.

(31) Nazar, L. F.; Koene, B. E.; Britten, J. F. Chem. Mater. 1996, 8 , 327.

(32) Whittingham, M. S. Chem. Rev. 2004, 104, 4271.

(33) Ban, C.; Chernova, N. A.; Whittingham, M. S. Electrochem. Commun. 2009, 11, 522.

(34) Mai, L. Q.; Chen, W.; Xu, Q.; Zhu, Q. Y. Microelectron Eng. 2003, 66, 199.

(35) Mai, L. Q.; Chen, W.; Xu, Q.; Zhu, Q. Y. Chem. Phys. Lett. 2003, 382, 307

(36) Mai, L. Q.; Chen, W.; Qi, Y. Y.; Dai, Y.; Jin, W. Solid State Phenomena 2007, 121-123, 789.

(37) Chan, C. K.; Peng, H.; Liu, G.; McIlwrath, K.; Zhang, X. F.; Huggins, R. A.; Cui, Y. Nat. Nanotechnol. 2008, 3, 31.

(38) Hosono, E.; Kudo, T.; Honma, I.; Matsuda, H.; Zhou, H. Nano Lett. 2009, 9, 1045.

(39) Chan, C. K.; Zhang, X. F.; Cui, Y. Nano Lett. 2008, 8, 307.

(40) Mai, L. Q.; Hu, B.; Chen, W.; Qi, Y. Y.; Lao, C. S.; Yang, R. S.; Dai, Y.; Wang, Z. L. Adv. Mater. 2007, 19, 3712.

(41) Mai, L. Q.; Hu, B.; Qi, Y. Y.; Dai, Y.; Chen, W. Int. J. Electrochem. Sci. 2008, 3, 216.

(42) Mai, L. Q.; Gao, Y.; Guan, J. G.; Hu, B.; Xu, L.; Jin, W. Int. J. Electrochem. Sci. 2009, 4, 755.

(43) Mai, L. Q.; Lao, C. S.; Hu, B.; Zhou, J.; Qi, Y. Y.; Chen, W.; Gu, E. D.; Wang, Z. L. J. Phys. Chem. B 2006, 110, 18138.

(44) Mai, L. Q.; Guo, W. L.; Hu, B.; Jin, W.; Chen, W. J. Phys. Chem. C 2008, 112, 423 .

(45) Chen, W.; Mai, L. Q.; Qi, Y. Y.; Jin, W.; Hu, T.; Guo, W. L.; Dai, Y.; Gu, E. D. Key Eng. Mater. 2007, 336-338, 2128.

(46) Zheng, L.; Xu, Y.; Jin, D.; Xie, Y. Chem. Mater. 2009, 21, 5681.
(47) Whittingham, M. S.; Dines, M. B. J. Electrochem. Soc. 1977, 124, 1387.

(48) Murphya, D. W.; Greenblatt, M.; Cava, R. J.; Zahurak, S. M. Solid State Ionics 1981, 5, 327.

(49) Li, L.; Pistoia, G. Solid State Ionics 1991, 47, 231.

(50) Li, L.; Pistoia, G. Solid State Ionics 1991, 47, 241.

(51) Jung, W. I.; Nagao, M.; Pitteloud, C.; Yamada, A.; Kann, R. J. Power Sources 2010, 195, 3328.

(52) Wang, S. T.; Zhang, Y. G.; Ma, X. C.; Wang, W. Z.; Li, X. B.; Zhang, Z. D.; Qian, Y. T. Solid State Commun. 2005, 136, 283.

(53) Bullard, J. W.; Smith, R. L. Solid State Ionics 2003, 160, 335.

(54) Chen, W.; Qi, Y. Y.; Mai, L. Q.; Xu, Q.; Liu, H. X.; Zhao, X. J. Hydrothermal Synthesis and Electrochemical Behavior of $\mathrm{MoO}_{3}$ Nanobelts for Lithium Batteries. In Proceedings of the 10th Asian conference on Solid State Ionics, the 10th Asian conference on Solid State Ionics, Sri Lanka, Jun12-16, 2006; Chowdari, B. V. R.; Careem, M. A.; Dissanayake, M. A. K. L.; Rajapakse, R. M. G.; Seneviratne, V. A.; Eds.; World Scientific Publishing: Singapore, 2006; pp: 833-840.

(55) Tsumura, T.; Inagaki, M. Solid State Ionics 1997, 104, 183.

(56) Subba, R. C.; Qi, Y.; Jin, W.; Zhu, Q.; Deng, Z.; Chen, W.; Mho, S. J. Solid State Electrochem. 2007, 11, 1239.

(57) Subba, R. C.; Walker, E. H., Jr.; Wen, C.; Mho, S. J. Power Sources 2008, 183, 330.

(58) Christian, P. A.; Carides, J. N.; DiSalvo, F. J.; Waszczak, J. V. J. Electrochem. Soc. 1980, 127, 2315.

(59) Chan, C. K.; Peng, H.; Twesten, R. D.; Jarausch, K.; Zhang, X. F.; Cui, Y. Nano Lett. 2007, 7(2), 490.

(60) Delmasa, C.; Cognac-Auradoua, H.; Cocciantellia, J. M.; Menetriera, M.; Doumerca, J. P. Solid State Ionics 1994, 69, 257.

(61) Garcia, B.; Millet, M.; Pereira-Ramos, J. P.; Baffier, N.; Bloch, D. J. Power Sources 1999, 81-82, 670.

(62) Lee, S. H.; Kim, Y. H.; Deshpande, R.; Parilla, P. A.; Whitney, E.; Gillaspie, D. T.; Jones, K. M.; Mahan, A.; Zhang, S.; Dillon, A. C. Adv. Mater. 2008, 20 (19), 3627.

(63) Huang, Y. H.; Goodenough, J. B. Chem. Mater. 2008, 20, 7237.

(64) Padhi, A. K.; Nanjundaswamy, K. S.; Goodenough, J. B. J. Electrochem. Soc. 1997, 144, 1188.

(65) Chen, J.; Vacchio, M. J.; Wang, S.; Chernova, N.; Zavalij, P. Y.; Whittingham, M. S. Solid State Ionics 2008, 178, 1676.

(66) Mai, L. Q.; Xu, L.; Han, C. H.; Xu, X.; Luo, Y. Z.; Zhao, S. Y.; Zhao, Y. L. Nano Lett. 2010, 10, 4750.

(67) Balke, N.; Jesse, S.; Morozovska, A. N.; Eliseev, E.; Chung, D. W.; Kim, Y.; Adamczyk, L.; García, R. E.; Dudney, N.; Kalinin, S. V. Nat. Nanotechnol. 2010, 5, 749 .

(68) Brezesinski, T.; Wang, J.; Tolbert, S. H.; Dunn, B. Nat. Mater. 2010, 9, 146 .

(69) Hu, Y. S.; Liu, X.; Müller, J. O.; Schlögl, R.; Maier, J.; Su, D. S. Chem. Int. Edit. 2009, 48, 210. 